\title{
Hydroxypropyl-beta and -gamma cyclodextrins rescue cholesterol accumulation in Niemann-Pick C1 mutant cell via lysosome-associated membrane protein 1
}

\author{
Ashutosh Singhal', Lajos Szente ${ }^{2}$, James E. K. Hildreth ${ }^{3}$ and Byeongwoon Song ${ }^{1}$
}

\begin{abstract}
Niemann-Pick type C (NPC) disease is a fatal hereditary neurodegenerative disorder characterized by a massive accumulation of cholesterol in lysosomes and late endosomes due to a defect in intracellular cholesterol trafficking. Dysfunction in intracellular cholesterol trafficking is responsible for about 50 rare inherited lysosomal storage disorders including NPC. The lysosomal proteins NPC1 and NPC2 play a crucial role in trafficking of cholesterol from late endosomes and lysosomes to other cellular compartments. However, the detailed mechanisms of cholesterol trafficking at the late endosomes/lysosomes (LE/LY) are poorly understood. Studies showed that 2-hydroxypropyl- $\beta$ cyclodextrin (HP $\beta C D$ ) alleviates the cholesterol accumulation defect in animal model and has been approved for a phase $2 \mathrm{~b} / 3$ clinical trial for NPC. HP $\beta C D$ is known to bind cholesterol; however, the mechanisms how HP $\beta C D$ mediates the exit of cholesterol from the LE/LY compartments are still unknown. Further, another cyclodextrin (CD) derivative, 2hydroxypropyl- $\gamma$-cyclodextrin (HPYCD), was shown to reduce intracellular cholesterol accumulation in NPC patient cells and NPC mice model. Herein, we identified a number of candidate proteins differentially expressed in NPC patientderived cells compared to cells derived from a healthy donor using a proteomic approach. Interestingly, both HP $\beta C D$ and HPYCD treatments modulated the expression of most of these NPC-specific proteins. Data showed that treatment with both CDs induces the expression of the lysosome-associated membrane protein 1 (LAMP-1) in NPC patientderived cells. Remarkably, LAMP-1 overexpression in HeLa cells rescued U18666A-induced cholesterol accumulation suggesting a role of LAMP-1 in cholesterol trafficking. We propose that HPßCD and HPYCD facilitate cholesterol export from the LE/LY compartments via the LAMP-1 protein, which may play a crucial role in cholesterol trafficking at the LE/ LY compartments when there is no functional NPC1 protein. Together, this study uncovers new cellular mechanisms for cholesterol trafficking, which will contribute to development of novel therapeutic approaches for lysosomal storage diseases.
\end{abstract}

\footnotetext{
Correspondence: Byeongwoon Song (bsong@mmc.edu)

'Department of Microbiology, Immunology, and Physiology, Meharry Medical College, Nashville, TN 37208, USA

${ }^{2}$ Cyclolab Cyclodextrin Research and Development Laboratory Ltd., H-1097

Budapest, Hungary

Full list of author information is available at the end of the article.

Edited by A. Finazzi-Agrò
}

\section{Introduction}

Lysosomes are acidic, membrane-bound organelles that play a crucial role in cholesterol metabolism. The majority of cellular demand for cholesterol is made through the receptor-mediated endocytosis of low-density lipoprotein (LDL) from plasma. Following an entry into cells, LDL is transported to the lysosomes via the endosomal

\section{(-) The Author(s) 2018}

\footnotetext{
(c) (i) Open Access This article is licensed under a Creative Commons Attribution 4.0 International License, which permits use, sharing, adaptation, distribution and reproduction cc) in any medium or format, as long as you give appropriate credit to the original author(s) and the source, provide a link to the Creative Commons license, and indicate if changes were made. The images or other third party material in this article are included in the article's Creative Commons license, unless indicated otherwise in a credit line to the material. If material is not included in the article's Creative Commons license and your intended use is not permitted by statutory regulation or exceeds the permitted use, you will need to obtain permission directly from the copyright holder. To view a copy of this license, visit http://creativecommons.org/licenses/by/4.0/.
} 
compartments. An acid lipase in the lumen of the lysosome hydrolyzes cholesteryl esters in $\mathrm{LDL}^{1}$, and the free cholesterol then exits the lysosomal compartment in order to reach other cellular compartments such as the plasma membrane and the endoplasmic reticulum (ER) where it performs both structural and regulatory roles ${ }^{2-4}$. A very little is known about how cholesterol is trafficked from lysosomes or late endosomes to other cellular organelles. Because late endosomes (LE) and lysosomes (LY) share many properties, we will henceforth refer to them indistinctively as either lysosomes or late endosomes/lysosomes (LE/LY).

Niemann-Pick type C (NPC) disease is a fatal hereditary disorder characterized by neurodegeneration, hepatosplenomegaly, and the accumulation of cholesterol and other lipids in lysosomes, and has implicated two lysosomal proteins NPC1 and NPC2 for the process of cholesterol exit from the LE/LY compartments ${ }^{5}$. Both proteins have cholesterol-binding property and homozygous mutations in either NPC1 or NPC2 cause lysosomal lipid accumulation and NPC disease, suggesting an essential role of these proteins in cholesterol export from the LE/LY compartments ${ }^{6}$.

Intracellular level and distribution of cholesterol is tightly regulated by receptor-mediated endocytosis of LDL, de novo biosynthesis, and intracellular trafficking between multiple organelles. Majority of the studies have focused on cholesterol uptake and biosynthesis; however, mechanisms of cholesterol trafficking towards the plasma membrane, plasma membrane to the ER via lysosomes, ER to the plasma membrane via lysosomes, and towards mitochondria are not well understood. Cholesterol levels in ER regulate de novo biosynthesis and uptake of cholesterol by controlling the proteolytic activation of the sterol regulatory element-binding proteins and the reesterification of cholesterol by the ER-resident acyl CoA: cholesterol acyltransferase. For use and storage at other cellular compartments, cholesterol has to exit the lysosomes. A model has been proposed for cholesterol trafficking at the LE/LY compartments, in which the NPC2 protein binds cholesterol in the lysosomal lumen and then transfer cholesterol to the $\mathrm{N}$-terminal domain of the lysosomal membrane-spanning NPC1 protein ${ }^{5}$. Cholesterol is then transported out of the LE/LY compartments by some unknown mechanisms. Cholesterol trafficking at the LE/LY compartments has gained interest recently due to its significance in understanding the mechanisms of NPC disease as well as other lysosomal storage disorders.

Cyclodextrins (CDs), a family of cyclic oligosaccharides consisting of $\alpha$-D-glucopyranose molecules joined by alpha-1-4-glycosidic linkages, can form complexes with a number of poorly water-soluble molecules including cholesterol through their hydrophobic cavity and enhance the solubility of the guest molecules via their hydrophilic outer surface ${ }^{7}$. Because of this cholesterol-binding property, selected $C D$ derivatives have been investigated as a potential therapy for NPC disease. One such CD, 2hydroxypropyl- $\beta$-cyclodextrin (HP $\beta C D)$, has been shown to alleviate the cholesterol accumulation defect in NPC1 mutant cells ${ }^{8}$. In preclinical studies, a direct brain injection of $\mathrm{HP} \beta C D$ into an NPC mouse model slowed the progression of neuronal loss and improved its survival ${ }^{9-11}$. Similar results were observed in a feline model of NPC disease, with an intra-cisternal administration conferring attenuated neurodegeneration and prolonged survival ${ }^{12}$. More recently, an intrathecal cyclodextrin injection in a single-NPC1 patient led to an increase in cholesterol redistribution in the central nervous system and improved vertical gaze palsy, a clinical indicator of NPC-linked neurodegeneration ${ }^{13}$. In 2013, due to the encouraging preclinical data, the HP $\beta C D$ entered into first-in-human clinical trials at the NIH Clinical Center. The HP $\beta C D$ successfully completed phase $1 / 2$ a clinical trial designed to test its safety and effectiveness. The study showed that intrathecal injections of $\mathrm{HP} \beta C D$ decreased the progression of neurological disease in NPC patients ${ }^{14}$. Based on the promising data of phase 1-2 clinical trial, currently, the $\mathrm{HP} \beta C D$ is being tested in a multicenter, multinational phase $2 \mathrm{~b} / 3$ clinical trial (NCT02534844). Although the efficacy of $\mathrm{HP} \beta C D$ in NPC disease is well established, the mechanism is still unknown. A recent study demonstrated that 2-hydroxypropyl- $\gamma$-cyclodextrin $(\mathrm{HP} \gamma \mathrm{CD})$ can reduce cholesterol accumulation in the NPC patient-derived cells and prolong survival in NPC model mice ${ }^{15}$. One study also showed that another $\mathrm{CD}$ derivative, methyl- $\beta$-cyclodextrin $(\mathrm{M} \beta \mathrm{CD})$, binds and activates AMP-activated protein kinase (AMPK), which was essential for $M \beta C D$-mediated reduction of cholesterol storage in NPC1 mutant cells ${ }^{16}$. This study provided the evidence of direct binding of $\mathrm{M} \beta \mathrm{CD}$ to the molecular target and reducing the cholesterol levels in NPC1 mutant cells. It is important to identify the molecular target of HP $\beta C D$ and HPyCD in order to develop drugs for NPC. We recently screened twelve derivatives of $\alpha, \beta$, and $\gamma$ CDs with different functional substitutions for their ability to modulate intracellular cholesterol accumulation; of the $12 \mathrm{CD}$ derivatives tested, $\mathrm{HP} \beta C \mathrm{CD}$ and $\mathrm{HP} \gamma \mathrm{CD}$ were able to rescue the cholesterol accumulation defect in NPC patient-derived cells $^{17}$. However, how HP $\beta C D$ or HPyCD triggers the cholesterol egress from the LE/LY compartments and normalize cellular cholesterol homeostasis is unanswered. Understanding these mechanisms will improve the cyclodextrin-based therapy and may reveal new drug targets for NPC disease. Therefore, it is important to understand the mechanisms by which the CDs rescue the intracellular cholesterol accumulation defect and to determine the cellular proteins or pathways that are critical for modulating intracellular cholesterol homeostasis. 
In the current studies, we used a proteomic approach to elucidate the mechanism of cyclodextrin-mediated exit of cholesterol from lysosomes. We identified a number of candidate proteins differentially expressed in NPC1 mutant cells compared to the wild-type cells; interestingly, $\mathrm{HP} \beta \mathrm{CD}$ and $\mathrm{HP} \gamma \mathrm{CD}$ were shown to modulate expression levels of most of these proteins. Through overexpression and knockdown approaches, we determined that the lysosome-associated membrane protein 1 (LAMP-1) mediates the cyclodextrin-induced exit of cholesterol from lysosomes when there is no functional NPC1 protein. We use these data to develop a hypothetical working model for cholesterol trafficking at the LE/ LY compartments in which the lysosomal membrane protein LAMP-1 substitutes the NPC1 protein and accepts cholesterol from the lysosomal luminal protein NPC2, prior to transferring cholesterol to other cellular compartments such as the ER membrane or plasma membrane.

\section{Results}

Effect of CDs on cell viability in fibroblasts derived from NPC patient and healthy donor

First, we tested the effect of various CD derivatives on cell viability (or metabolic activity) in human fibroblasts from a healthy donor or from a NPC patient with a defect in NPC1 gene. The CDs tested include the native forms of $\alpha-, \beta-$, and $\gamma-C D$ s as well as their chemical modifications with hydroxypropyl (HP), randomly methylated (RM), or carboxymethyl (CM) group. Figure 1 shows that $1 \mathrm{mM}$ concentration of all of the $\mathrm{CD}$ derivatives did not change cell viability (as depicted by metabolic activity). However, at 5 or $10 \mathrm{mM}$ concentration, native or RM-modified $\alpha$ and $\beta-C D$ treatment significantly decreased the metabolic activity in both cell types (Fig. 1a, b, d, e), but $\gamma$-CDs did not affect cell viability (Fig. 1c, f). Interestingly, substitutions with carboxymethyl or hydroxypropyl group in $\alpha$ CDs and $\beta$-CDs significantly abrogated their toxicity, as $C M-\alpha-C D$ and $C M-\beta-C D$ did not alter metabolic activity at $5 \mathrm{mM}$ or $10 \mathrm{mM}$ concentration in both cell types. Modification of $\alpha$-, $\beta$ - or $\gamma$-CD with hydroxypropyl group did not show any toxicity in both cell types except that only slightly decreased metabolic activity was observed in cells treated with $\mathrm{HP}-\gamma-\mathrm{CD}$ at 5 or $10 \mathrm{mM}$ concentration. Modification of $\alpha$ - or $\beta-C D$ with methyl group did not show toxicity at $5 \mathrm{mM}$ concentration, but methylated $\alpha$ $\mathrm{CD}$ and $\beta-C D$ significantly decreased metabolic activity at $10 \mathrm{mM}$ concentration. Modification of $\gamma$-CD with methyl group slightly decreased metabolic activity at 5 or $10 \mathrm{mM}$ in both cell types. Together, these data suggest that neither native $\mathrm{CDs}$ nor any $\mathrm{CD}$ derivatives are toxic at $1 \mathrm{mM}$ concentration in healthy as well as $N P C 1^{-1}$ fibroblasts and therefore, we selected this concentration for our study.

\section{Effects of CDs on lysosomal accumulation of cholesterol}

We examined the effects of the $\mathrm{CD}$ derivatives on the levels of free cholesterol in lysosomes in NPC1 mutant fibroblast cells using filipin, a fluorescent compound that specifically binds to unesterified cholesterol present in lysosomes. As depicted in microscope image, NPC1 mutant cells (Fig. 2e) showed a robust accumulation of cholesterol as compared to healthy skin fibroblast cells (Fig. 2a) reflecting a defect in cholesterol exit from lysosomes. Interestingly, $\mathrm{HP} \beta \mathrm{CD}$ or $\mathrm{HP} \gamma \mathrm{CD}$ treatment remarkably reduced cholesterol levels in NPC1 mutant cells (Figure $2 \mathrm{~g}, \mathrm{~h}$ ) whereas $\mathrm{HP \alpha CD}$ did not affect the intracellular cholesterol accumulation (Figure 2f). Therefore, we have selected $\mathrm{HP} \beta C D$ and $\mathrm{HP \gamma CD}$ to further investigate the molecular mechanisms by which these CDs facilitate cholesterol exit from lysosomes and reduce the cholesterol levels in NPC1 mutant cells.

\section{Effects of HPBCD and HPyCD treatments on the expression of cellular proteins}

To understand the potential mechanisms of HP $\beta C D$ - or HPyCD-mediated modulation in intracellular cholesterol homeostasis, we attempted to identify differentially expressed proteins involved in cholesterol metabolism and trafficking. We utilized automated multidimensional protein identification technology (MudPit), which is a shotgun proteomics approach usually applied to obtain overviews of protein expression in biological samples ${ }^{18}$. We applied standard label-free quantification of high mass resolution LC-MS data ${ }^{19}$ for high throughput analysis of protein expression in NPC1 mutant cells. LC-MS/ MudPIT analysis showed a robust change in protein expression profile in NPC patient-derived cells compared to cells derived from a healthy donor, which is modulated remarkably, when NPC patient-derived cells were subjected to HP $\beta C D$ or $\mathrm{HP} \gamma \mathrm{CD}$ treatment. First, we compared protein levels between healthy donor-derived cells (the wild-type control) and NPC patient-derived cells (NPC1 mutant) and identified 46 differentially expressed proteins (annotated by protein database) whose levels differed more than twofold in NPC1 mutant cells compared to wild-type cells (Table 1). These proteins may potentially be implicated in the control of cholesterol homeostasis in normal cells with abnormal expression of these proteins resulting in the cholesterol trafficking defect in NPC1 mutant cells (Fig. 3 and Table 1). The modulation of these proteins might be essential to correct the cholesterol trafficking defect in NPC1 mutant cells. Remarkably, 35 proteins out of the these 46 proteins were altered more than twofold in NPC1 mutant cells upon treatment with $\mathrm{HP} \beta C D$ or $\mathrm{HP \gamma CD}$, with their levels being shifted toward those of healthy donor cells from those of NPC patient cells (Fig. 3). These 35 proteins include: 12 proteins upregulated by both $\mathrm{HP} \beta C D$ and $\mathrm{HP \gamma CD}, 5$ 


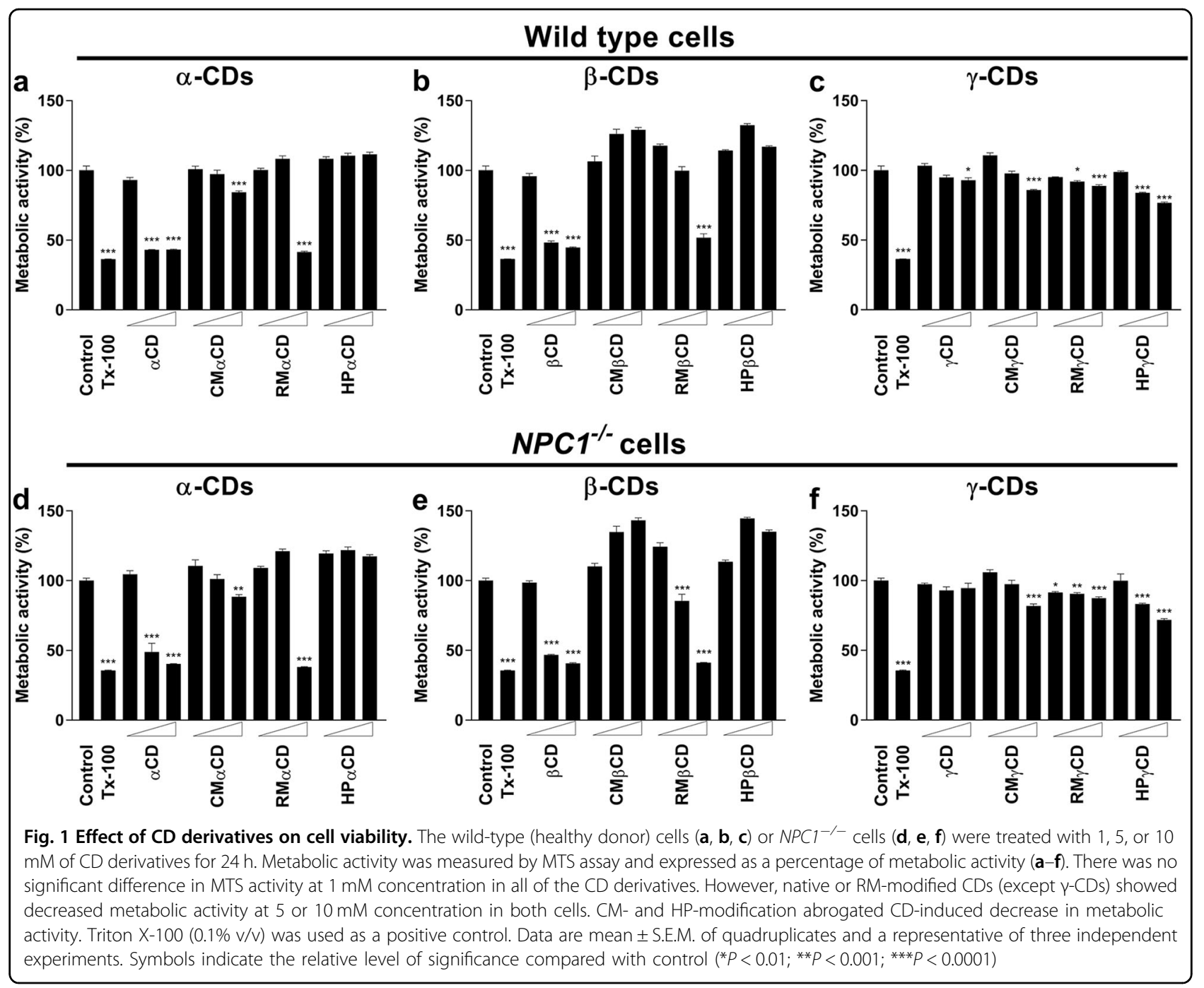

proteins upregulated by $\mathrm{HP} \beta \mathrm{CD}$ only, 1 protein upregulated by $\mathrm{HP \gamma CD}$ only, 10 proteins downregulated by both $\mathrm{HP} \beta C D$ and $\mathrm{HP \gamma CD}, 3$ proteins downregulated by $\mathrm{HP} \beta C D$ only, and 4 proteins downregulated by $\mathrm{HP} \gamma \mathrm{CD}$ only. We hypothesized that some of these 35 proteins may play a critical function in $\mathrm{CD}$-mediated modulation of the intracellular cholesterol trafficking. Though, the MudPIT technique is fast and sensitive with good reproducibility, it lacks the ability to provide quantitative information and relies on label-free quantification method ${ }^{20-22}$. Therefore, we validated the quantitative information of our selected proteins with other methods such as western blots. There were 137 or 172 proteins differentially expressed in NPC1 mutant cells upon treatment with $\mathrm{HP} \beta \mathrm{CD}$ or $\mathrm{HP \gamma CD}$, respectively, when compared to untreated cells (Supplemental Table 1). Interestingly, majority of the differentially expressed proteins were common in HP $\beta C D$ and HPyCD treated cells. A total 93 proteins were common for $\mathrm{HP} \beta C D$ and $\mathrm{HP} \gamma \mathrm{CD}$ treatment and 44 proteins were differentially expressed exclusively in $\mathrm{HP} \beta C D$ treated cells and 72 proteins were differentially expressed in $\mathrm{HP} \gamma \mathrm{CD}$ treated cells (Supplemental Table 1). While we focus on the role of one candidate protein in this study, we can not rule out the involvement of several other proteins in the regulation of cellular cholesterol homeostasis.

\section{LAMP-1 expression and lysosome distribution in NPC1 mutant cells}

Out of 35 candidate proteins differentially expressed in $N P C 1$ mutant cells treated with HP $\beta C D$ or HPYCD, we selected the LAMP-1 protein to further investigate its role in cholesterol trafficking at the LE/LY compartments. According to MudPit data, the LAMP-1 protein was upregulated by $\mathrm{HP} \beta C D$ and $\mathrm{HP \gamma CD}$ in $N P C 1$ mutant cells. Therefore, we verified this finding by Western blotting (Fig. 4a), which shows that the lysosomal membrane protein LAMP-1 was upregulated by HP $\beta C D$ and HPYCD consistent with the MudPit data. However, 


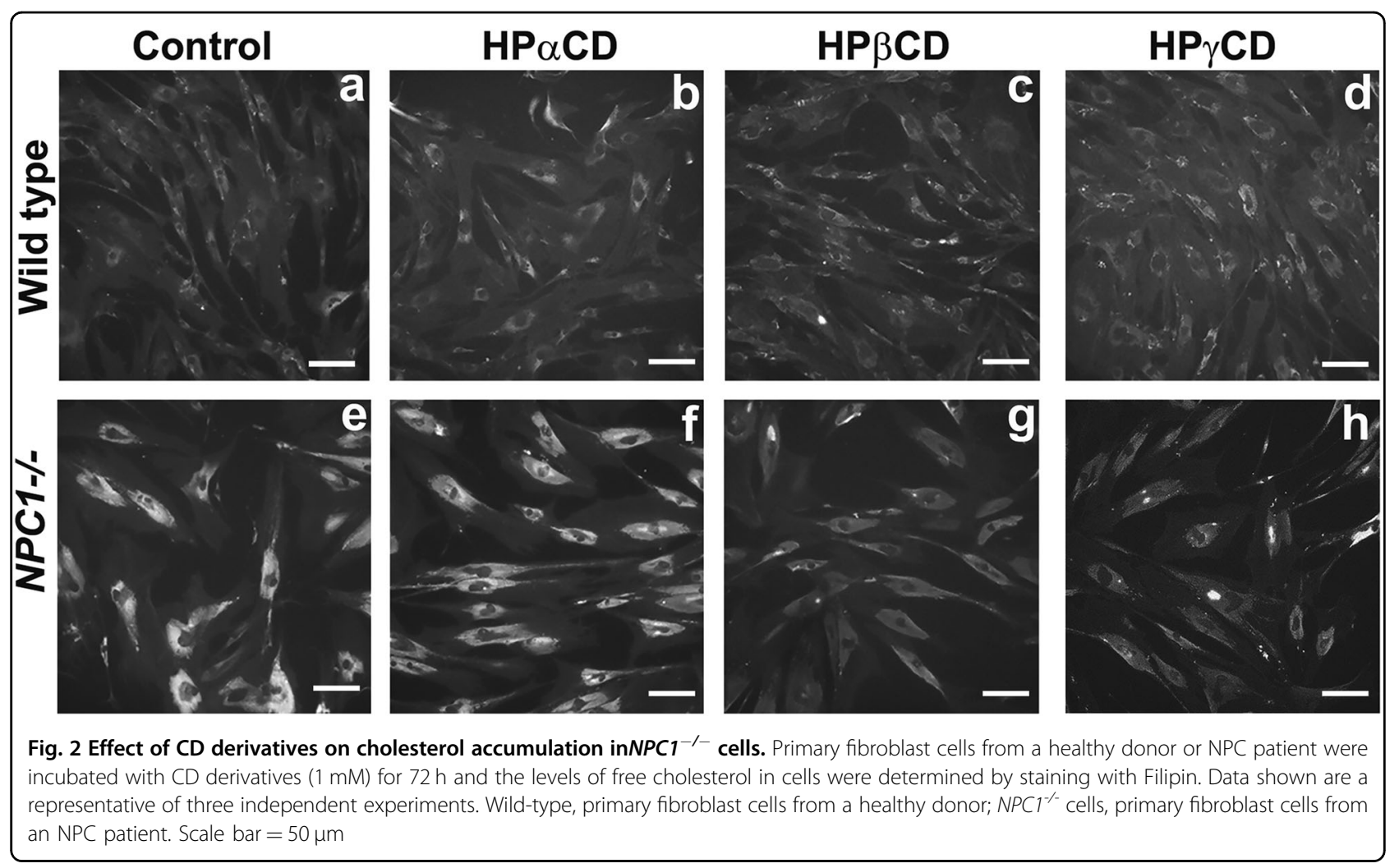

LAMP-2 and LAMP-3 did not show any change in expression in NPC1 mutant cells after HP 3 CD or HPYCD treatment (Fig. 4b).

As LAMP-1 is a marker of lysosomes, we determined whether lysosomal positioning is altered upon treatment with $\mathrm{HP} \beta \mathrm{CD}$ or $\mathrm{HP} \gamma \mathrm{CD}$. Interestingly, lysosomes were distributed more widely within the cell upon $\mathrm{HP} \beta C D$ or $\mathrm{HP}$ YCD treatment in NPC1 mutant cells whereas the lysosomes were clustered near the cell center in untreated NPC1 mutant cells (Fig. 4c, d). These results are consistent with previous studies demonstrating that cholesterol accumulation or NPC1 deficiency is associated with a defect in LE/LY mobility ${ }^{23-25}$, and suggest that cholesterol trafficking at the LE/LY compartments may be important for maintaining the biogenesis, distribution, and function of lysosomes.

\section{Role of LAMP-1 in cholesterol exit from lysosomes}

To understand whether the LAMP-1 protein has any role in CD-mediated cholesterol exit from lysosomes in NPC1 mutant cells, we made two stable cell lines of HeLa using a lentivirus-based plasmid vector system. HeLa cells either overexpressing the LAMP-1 protein (Hela-LE) or stably carrying shRNA specific for LAMP-1 (Hela LAMP1 knockdown or HeLa-Lkd) showed significantly increased or decreased levels of LAMP-1, respectively, as compared to cells transfected with a negative control plasmid (Fig. 5a). Both HeLa-LE and HeLa-Lkd cell lines did not show any sign of cytotoxicity due to overexpression or knockdown of LAMP-1 (Fig. 5b, c). We used U18666A, an inhibitor of cellular cholesterol trafficking that induces an NPC1 mutation phenotype, to investigate the role of the LAMP-1 protein in cholesterol trafficking at the LE/LY compartments. Treatment with U18666A ( $5 \mu \mathrm{g} / \mathrm{ml}$ for 3 days) did not alter the metabolic activity of any of the HeLa cell lines (Fig. 5b, c). Filipin imaging showed that U18666A treatment significantly increased cholesterol accumulation in control HeLa cells (Fig. 5d, left). Interestingly, LAMP-1 overexpression rescued the cholesterol accumulation defect in HeLa cells (Fig. 5d, middle) whereas LAMP-1 knockdown did not alleviate the cholesterol accumulation defect (Fig. 5d, right). Moreover, LAMP-1 knockdown caused a slight increase in the levels of cholesterol compared to the control cells upon treatment with U18666A. Taken together, these data suggest that the LAMP-1 protein plays an important role in cholesterol trafficking at the LE/LY compartments, and potentially re-positions the lysosomes toward other organelles and/or the plasma membrane in order to maintain cholesterol homeostasis.

\section{Discussion}

Herein, we have demonstrated that $\mathrm{HP} \beta \mathrm{CD}$ and $\mathrm{HP} \gamma \mathrm{CD}$ upregulate the expression of the lysosomal membrane 
Table 1 Neimann-Pick disease type C1-specific proteins that are differentially expressed by treatment with HPßCD or HPYCD. LAMP-1 protein was identified as significantly upregulated upon HPYCD treatment (also upon HP $\beta C D$ treatment when compared with the NPC group) as compared to either healthy or NPC control cells

\begin{tabular}{|c|c|c|c|c|c|}
\hline Accession ID & Protein ID & Name of the protein & $\begin{array}{l}N P C 1^{-1-} \\
\text { Con }\end{array}$ & $\begin{array}{l}N P C 1^{-/-} \\
+\mathrm{HP} \beta C D\end{array}$ & $\begin{array}{l}\mathrm{NPC1}^{-/-} \\
+\mathrm{HP} \gamma \mathrm{CD}\end{array}$ \\
\hline NP_001276332 & MMS19 & MMS19 homolog, cytosolic iron-sulfur assembly component & 9.35 & 176.17 & 3.92 \\
\hline XP_005246524 & GLS & Glutaminase & 0.2 & 2.08 & 0.47 \\
\hline NP_001340 & DARS & Aspartyl-tRNA synthetase & 115.28 & 43.37 & 185.52 \\
\hline XP_011529432 & FLNA & Filamin A & 0.43 & 0.24 & 0.07 \\
\hline NP_005336 & HSPA1A & Heat shock protein family $\mathrm{A}(\mathrm{Hsp} 70)$ member $1 \mathrm{~A}$ & 4.01 & 0.98 & 14.89 \\
\hline NP_002878 & RARS & Arginyl-tRNA synthetase & 0.04 & 1.61 & 3.26 \\
\hline NP_001030168 & RPL14 & Ribosomal protein L14 & 5.59 & 0.18 & 0.01 \\
\hline XP_005267356 & AHNAK2 & AHNAK nucleoprotein 2 & 0.36 & 0.38 & 0.07 \\
\hline NP_004795 & $\mathrm{CIAO} 1$ & Cytosolic iron-sulfur assembly component 1 & 0.03 & 0.38 & 1.08 \\
\hline NP_001814 & CKB & Creatine kinase $B$ & 2.15 & 2.51 & 0.13 \\
\hline XP_011533095 & GOLGA3 & Golgin A3 & 0.05 & 1.72 & 3.89 \\
\hline NP_000975 & RPL23A & Ribosomal protein L23a & 0.47 & 0.85 & 0.07 \\
\hline NP_006504 & SARS & Seryl-tRNA synthetase & 2.59 & 3.42 & 0.42 \\
\hline NP_037466 & GMPPB & GDP-mannose pyrophosphorylase B & 0.03 & 0.04 & 0.97 \\
\hline NP_002619 & PFN2 & Profilin 2 & 0.31 & 0.25 & 0.05 \\
\hline NP_001287921 & EIF3K & Eukaryotic translation initiation factor 3 subunit $\mathrm{K}$ & 7.26 & 3.65 & 0.34 \\
\hline NP_006657 & RUVBL2 & RuvB like AAA ATPase 2 & 7.17 & 1.38 & 0.34 \\
\hline NP_001011 & RPS16 & Ribosomal protein S16 & 0.1 & 0.27 & 0.6 \\
\hline NP_000980 & RPL30 & Ribosomal protein $\mathrm{L} 30$ & 13.34 & 3.56 & 9.95 \\
\hline NP_001687 & ATP6V1E1 & ATPase $\mathrm{H}+$ transporting $\mathrm{V} 1$ subunit $\mathrm{E} 1$ & 0.5 & 0.43 & 0.02 \\
\hline NP_001419 & ENO1 & Enolase 1 & 0.05 & 1.29 & 0.27 \\
\hline NP_009057 & VCP & Valosin-containing protein & 2.51 & 2.35 & 4.89 \\
\hline NP_002583 & PCNA & Proliferating cell nuclear antigen & 0.5 & 8.3 & 0.08 \\
\hline NP_579899 & MYOF & Myoferlin & 34.77 & 10.59 & 4.08 \\
\hline NP_006127 & CAPZA2 & Capping actin protein of muscle Z-line alpha subunit 2 & 0.21 & 0.11 & 0.22 \\
\hline NP_001018083 & PCK2 & Phosphoenolpyruvate carboxykinase 2, mitochondrial & 61.89 & 19.43 & 3.63 \\
\hline NP_001279 & CLIC1 & Chloride intracellular channel 1 & 0.03 & 5.21 & 2.19 \\
\hline NP_067072 & SNX6 & Sorting nexin 6 & 0.03 & 2.06 & 0.04 \\
\hline NP_066977 & PSAT1 & Phosphoserine aminotransferase 1 & 0.03 & 3.64 & 0.31 \\
\hline NP_001075109 & PRKDC & Protein kinase, DNA-activated, catalytic polypeptide & 0.26 & 0.07 & 0.01 \\
\hline NP_000393 & G6PD & Glucose-6-phosphate dehydrogenase & 0.01 & 2.15 & 0.84 \\
\hline NP_006182 & PA2G4 & Proliferation-associated 2G4 & 3.66 & 0.2 & 1.74 \\
\hline XP_005253351 & STRAP & Serine/threonine kinase receptor associated protein & 0.16 & 2.51 & 0.68 \\
\hline NP_001257356 & PSMD5 & Proteasome $26 \mathrm{~S}$ subunit, non-ATPase 5 & 0.35 & 0.77 & 0.02 \\
\hline NP_055416 & EHD2 & EH domain containing 2 & 3.87 & 0.1 & 0.25 \\
\hline NP_056991 & LAP3 & Leucine aminopeptidase 3 & 2.25 & 1.28 & 6.33 \\
\hline NP_001185709 & ARPC4 & Actin related protein $2 / 3$ complex subunit 4 & 2 & 0.08 & 0.78 \\
\hline
\end{tabular}


Table 1 continued

\begin{tabular}{|c|c|c|c|c|c|}
\hline Accession ID & Protein ID & Name of the protein & $\begin{array}{l}N P C 1^{-1-} \\
\text { Con }\end{array}$ & $\begin{array}{l}N P C 1^{-/-} \\
+\mathrm{HP} \beta C D\end{array}$ & $\begin{array}{l}N P C 1^{-1-} \\
+\mathrm{HP} Y \mathrm{CD}\end{array}$ \\
\hline NP_001257411 & PSMD11 & Proteasome 265 subunit, non-ATPase 11 & 0.07 & 0.82 & 0.01 \\
\hline XP_011523798 & NPEPPS & Aminopeptidase puromycin sensitive & 0.4 & 0.01 & 0.03 \\
\hline NP_001137290 & SERPINB2 & Serpin family B member 2 & 16.62 & 25.23 & 8.1 \\
\hline NP_620164 & CMBL & Carboxymethylenebutenolidase homolog & 0.43 & 0.01 & 0.02 \\
\hline NP_001121188 & ETFA & Electron transfer flavoprotein alpha subunit & 2.05 & 11.03 & 3.67 \\
\hline NP_004981 & MARS & Methionyl-tRNA synthetase & 8.58 & 1.22 & 0.3 \\
\hline NP_002787 & PSMB4 & Proteasome subunit beta 4 & 78.05 & 21.33 & 9.59 \\
\hline NP_003133 & SSB & Sjogren syndrome antigen B & 0.15 & 1.19 & 0.17 \\
\hline NP_005552 & LAMP1 & Lysosomal associated membrane protein 1 & 0.43 & 1.69 & 3.71 \\
\hline
\end{tabular}

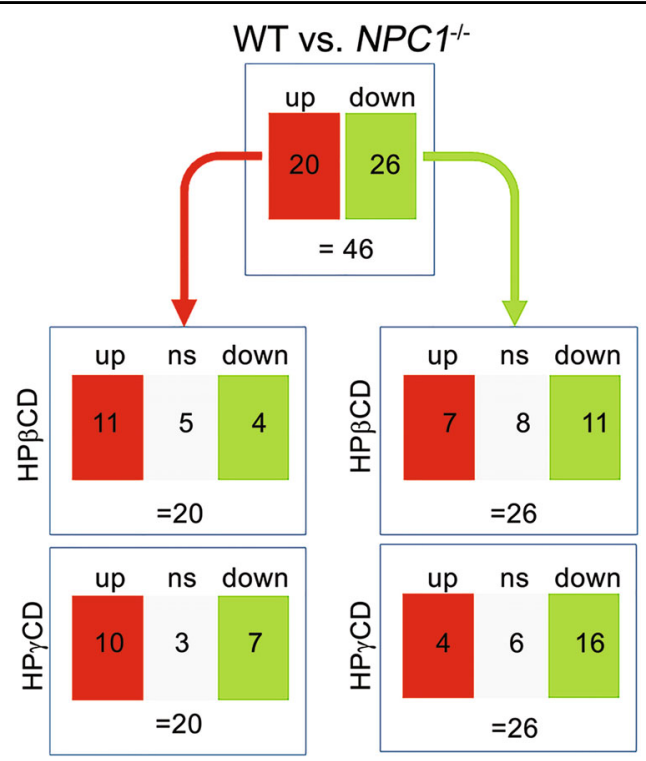

Fig. 3 Neimann-Pick disease type C 1-specific and cyclodextrinregulated proteins identified from a mass-spectrometric platform. LC-MS proteomic analysis of NPC patient-derived fibroblasts that were treated with HPBCD or HPYCD. Cell lysates were prepared from NPC patient-derived fibroblasts or primary fibroblast cells from a healthy donor after treatment with HPBCD or HPYCD (1 $\mathrm{mM}, 72 \mathrm{~h}$ ). Protein samples were subjected to LC-MS analysis (MudPIT) and peptide identification using PEAKS8.0 and MyriMatch. Untreated fibroblasts from an NPC patient and untreated fibroblasts from a healthy donor were included as controls. Four groups of samples were analyzed; untreated healthy donor cells (Healthy), untreated NPC patient cells (NPC), NPC patient cells treated with HPBCD (NPC/ $\mathrm{HP} \beta C D$ ), and NPC patient cells treated with HPYCD (NPC/HPyCD). The flow chart shows a total 46 differentially expressed proteins in $\mathrm{NPC1}^{-1}$ cells compared to that of cells from a healthy donor (top). HP $\beta C D$ (middle) or HPYCD (bottom) treatment modulated expression levels of these proteins. A pairwise comparison was performed between WT vs. $N P C ; W T$ vs. NPC + HPßCD; WT vs. NPC + HPץCD protein LAMP-1 and facilitate cholesterol trafficking at the LE/LY compartments, rescuing the cholesterol accumulation defect in NPC patient-derived fibroblast cells (NPC1 mutant). By using a proteomic approach, we identified a number of proteins that may potentially be implicated in cholesterol metabolism or trafficking. It is very well established that $\mathrm{HP} \beta C D$ (and possibly $\mathrm{HP} \gamma \mathrm{CD}$ ) modulates intracellular cholesterol accumulation and alleviates NPC disease ${ }^{5,10,15,26,27}$. However, the molecular mechanisms by which $\mathrm{HP} \beta C D$ or $\mathrm{HP} \gamma \mathrm{CD}$ facilitates the exit of cholesterol from lysosomes and reduces the intracellular cholesterol accumulation in NPC1 mutant cells are largely unknown. Our data showed that both $\mathrm{HP} \beta C D$ and $\mathrm{HP} \gamma \mathrm{CD}$ rescue the cholesterol accumulation defect in NPC1 mutant cells (Fig. 2), despite the differences in their physicochemical properties ${ }^{17}$. While $\mathrm{HP} \gamma \mathrm{CD}$ has a much weaker capacity to solubilize or extract cholesterol compared to $\mathrm{HP} \beta C D^{17,28}, \mathrm{HP} \gamma \mathrm{CD}$ was very effective in alleviating the cholesterol accumulation defect in a cell culture model or mouse model of NPC disease $\mathrm{s}^{15,17}$. The view that the CDs alleviate the cholesterol accumulation defect in NPC simply by binding and/ or extracting cholesterol should be re-evaluated. Together, these findings suggest that CD-mediated modulation of intracellular cholesterol accumulation may involve a multitude of mechanisms ${ }^{17}$. We demonstrated that treatment with $\mathrm{HP} \beta \mathrm{CD}$ or $\mathrm{HP} \gamma \mathrm{CD}$ induces changes in the expression of a number of proteins (Table 1) involved in diverse cellular pathways such as intracellular vesicle transport, lipid metabolism, and cell growth, etc. Further study is warranted to better understand the functional significance of the cellular signaling pathways and proteins that are induced by HP $\beta C D$ and HP $\gamma C D$.

The lysosomal membrane glycoproteins LAMP-1 and LAMP-2 are the two most abundant proteins in the lysosome lining the limiting membrane ${ }^{29}$. The LAMP-1 and LAMP-2 proteins are 37\% identical and comprised of 


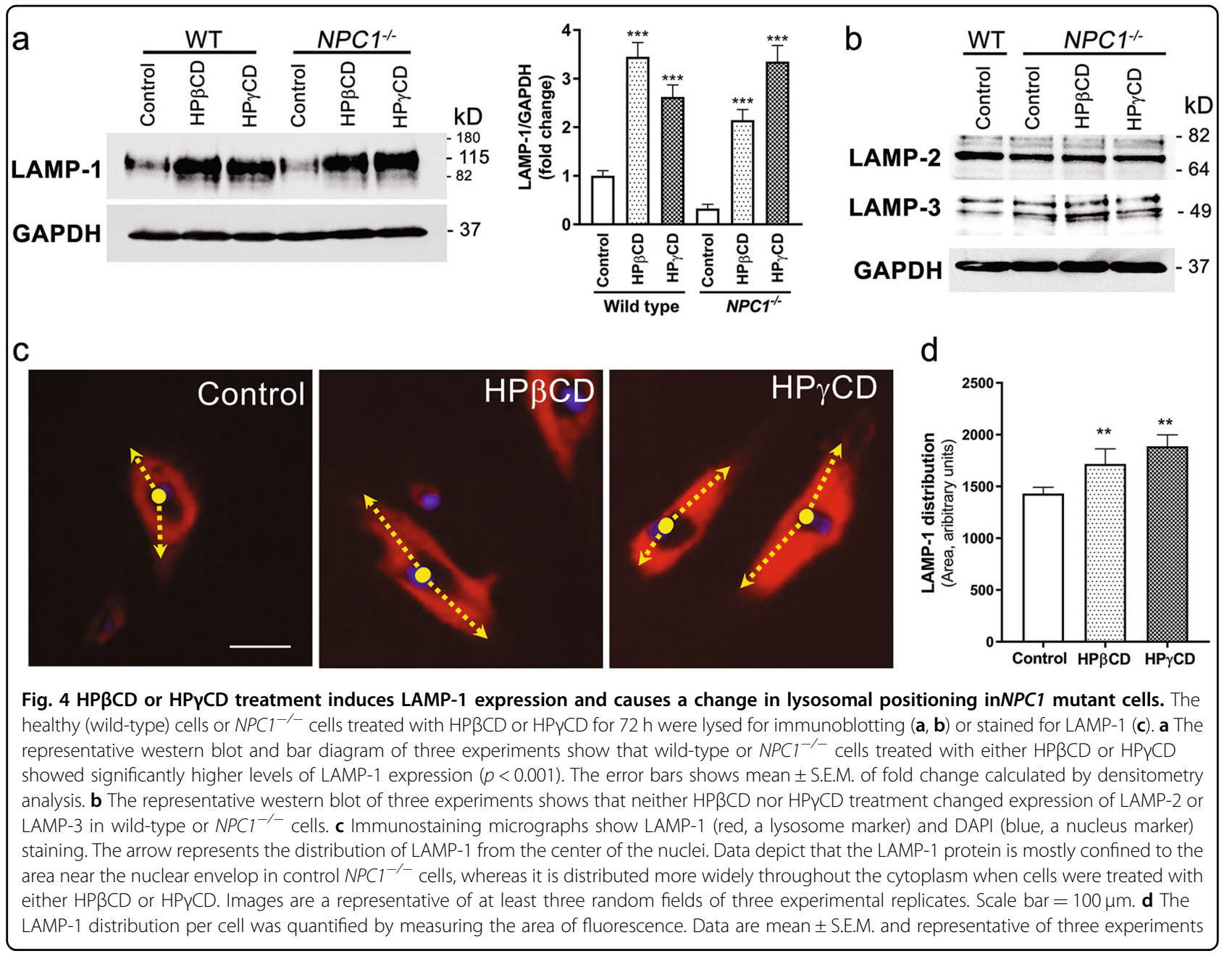

a short cytoplasmic domain, a single transmembrane domain, and a highly, N- and O-glycosylated luminal domain, and may have overlapping functions ${ }^{30-32}$. However, relatively little is known about the functions of these proteins in cholesterol homeostasis and they are often presumed to be structural components. One study showed that LAMPs are required for fusion of lysosomes with phagosomes ${ }^{33}$. Recently, the role of LAMPs in cholesterol homeostasis has gained interest. LAMP-2deficient cells showed cholesterol accumulation, which was corrected by LAMP-2 expression ${ }^{34,35}$. A proteomic study identified LAMP-1 and LAMP-2 as potential cholesterol-binding proteins ${ }^{36}$. It has been suggested that the LAMP-1/-2 proteins can bind cholesterol directly and perhaps facilitate cholesterol export from lysosomes ${ }^{37}$. Despite these clues, the detailed functions of LAMP-1 in cholesterol trafficking have remained unclear.

In this study, we demonstrated that LAMP-1 overexpression can rescue pharmacologically (U18666A) induced cholesterol accumulation in HeLa cells, a mimic of NPC1 or NPC2 mutation. However, LAMP-1 knockdown did not induce cholesterol accumulation in untreated HeLa cells, which have functional NPC1 and NPC2 proteins. These results suggest that there is a functional redundancy between LAMP-1 and NPC1/ NPC2 proteins for the regulation of cholesterol homeostasis. Based on these data, we speculate that in some circumstances, especially in the absence of a functional NPC1 protein, LAMP-1 is capable of binding cholesterol and directing it to the lysosomal membrane for trafficking to the plasma membrane and/or the ER. If this hypothesis is true, then NPC1 mutant cells should have higher LAMP-1 expression levels than the control wild-type cells to maintain intracellular cholesterol homeostasis. In contrast, we observed the opposite results; NPC1 mutant cells showed lower levels of LAMP-1 compared to the wild-type control cells. Considering that our observation is based on only one cell line, further investigations are warranted (including more cell lines) to confirm this possibility. It seems that an alteration in the levels of intracellular cholesterol does not directly provide a signal for the cellular machinery to upregulate LAMP-1 


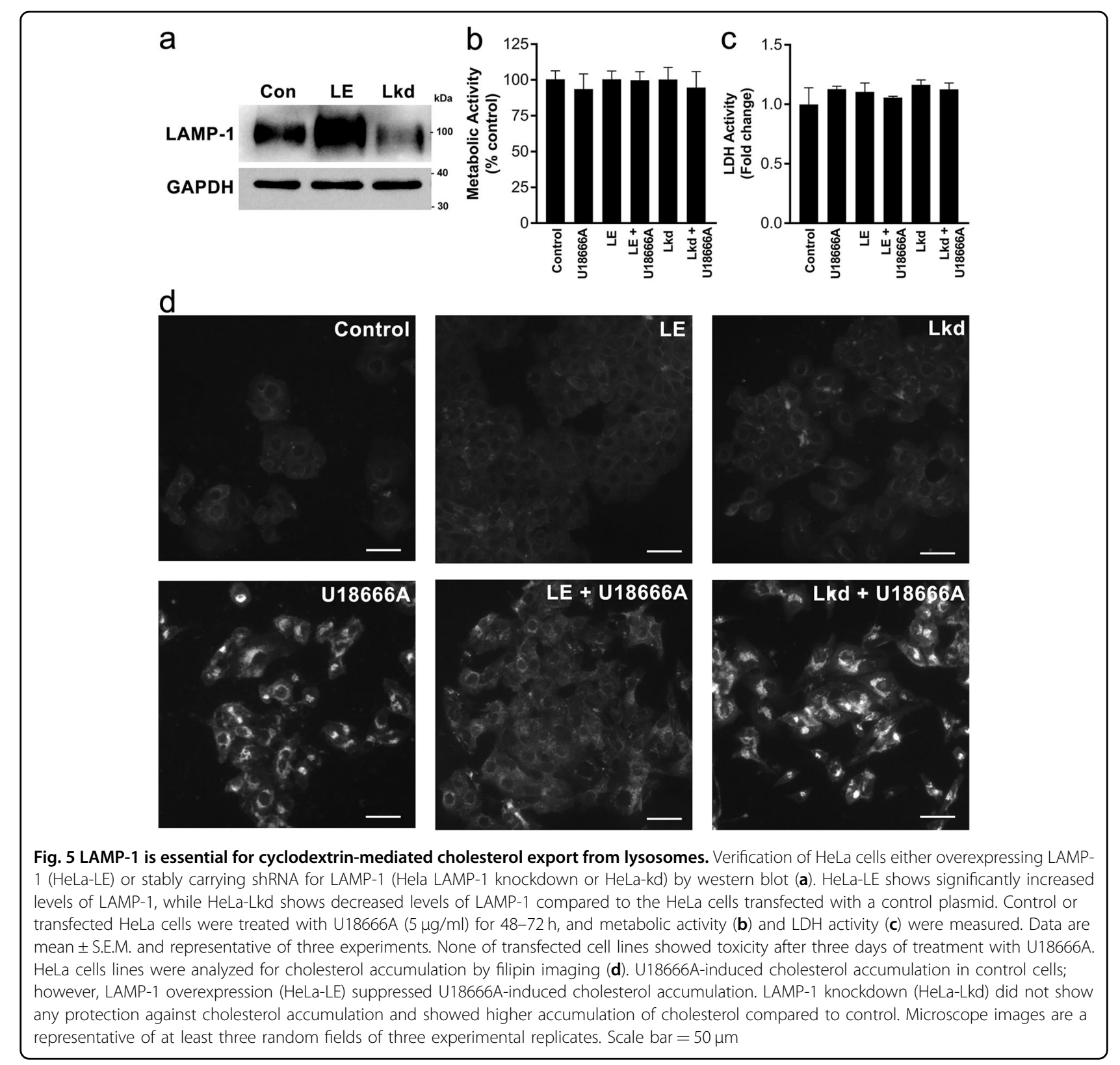

expression in order to maintain cholesterol homeostasis in NPC1 mutant cells. In this scenario, treatment with cyclodextrins ( $\mathrm{HP} \beta C \mathrm{CD}$ and $\mathrm{HP} \gamma \mathrm{CD})$ has more significance for inducing LAMP-1 expression in NPC1 mutant cells (which maintain low levels of LAMP-1 otherwise) to restore cholesterol homeostasis. Therefore, this study found a new role of LAMP-1 in intracellular cholesterol homeostasis as well as the potential for the cyclodextrinbased therapeutic strategies for NPC disease.

One of the major unanswered questions in cellular cholesterol homeostasis is how free cholesterol exits the LE/LY compartments and reaches other cellular compartments such as the ER and the plasma membrane. It was suggested that the lysosomal luminal protein NPC2 binds cholesterol released from LDL and hands-off to the $\mathrm{N}$-terminal domain of the lysosomal membrane-spanning protein NPC1, which then inserts the cholesterol into the lysosomal membrane ${ }^{5}$. Crystallography, genetic and in vitro binding studies suggested that NPC2 and NPC1 bind to cholesterol in opposite orientation such that NPC2 exposes the $3 \beta-\mathrm{OH}$ group and buries the isooctyl side chain deep within a hydrophobic pocket; however, when NPC1 binds cholesterol, it exposes the isooctyl side chain and buries the $3 \beta-\mathrm{OH}$ group ${ }^{38,39}$. This change in the orientation of cholesterol during its transfer from NPC2 to NPC1 appears to allow NPC1 to insert the isooctyl side chain of cholesterol into the lysosomal membrane prior to trafficking to other cellular compartments. Because 


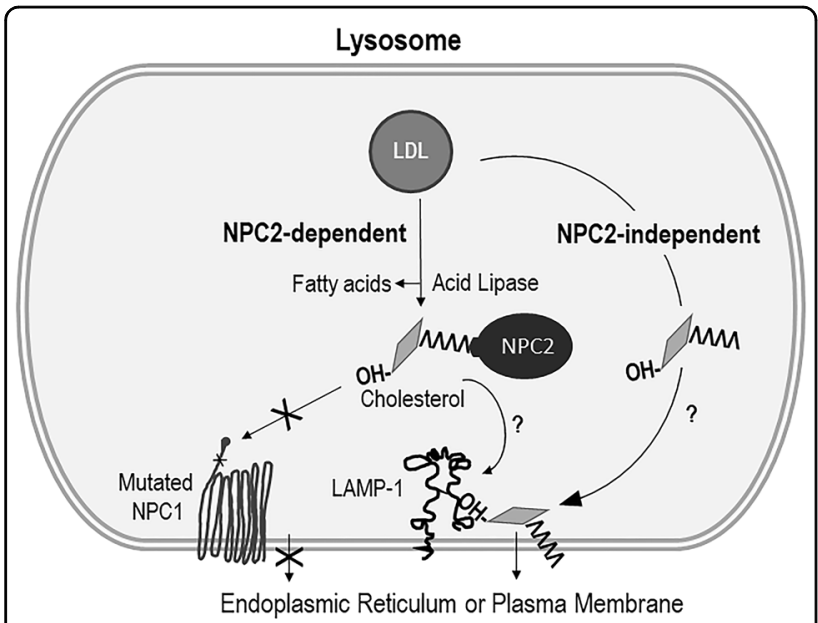

Scheme 1 A hypothetical model of cholesterol egress from lysosome to ER or PM in NPC1 mutant cells. When NPC1 mutant cells are treated with $H P \beta C D$ or $H P \gamma C D$, free cholesterol released from LDL is handed-off to LAMP-1 by NPC2 in an NPC2-dependent manner or it directly bind to LAMP-1

LAMP-1 is known to bind NPC1, NPC2, and cholesterol $^{37}$, it will be very interesting to study how LAMP-1 is involved in the process of cholesterol exit from the LE/LY compartments. Further studies are necessary to understand whether cholesterol is channeled to LAMP-1 directly or cholesterol is handed-off to LAMP-1 through NPC2 or NPC1 prior to the exit from the LE/LY compartments.

It is possible that the orientation of cholesterol-binding to LAMP-1 is similar to NPC1 rather than to NPC2 (Scheme 1). LAMP-1 is a large lysosomal membrane glycoprotein, whereas NPC2 is a relatively small protein present in the lumen of lysosomes ${ }^{40}$. Moreover, NPC2 alone is not sufficient for cholesterol egress from lysosomes, and NPC1 is essential for this process ${ }^{6,41}$. Our data suggest that the LAMP-1 protein is important in maintaining cholesterol trafficking at the LE/LY compartments, in particular when NPC1 function is absent or limited. Together, these findings support a novel role of LAMP-1 in cyclodextrin-mediated cholesterol homeostasis. However, this hypothesis needs further investigations and will be the focus of our future studies.

\section{Materials and methods Reagents}

Cell culture media and reagents were purchased from Thermo Fisher Scientific (Waltham, MA, USA); these include Dulbecco's modified Eagle's medium (DMEM), MEM non-essential amino acids, fetal bovine serum (FBS), penicillin, and streptomycin. A CellTiter 96 Aqueous One Solution Cell Proliferation Assay System was purchased from Promega (Madison, WI, USA). An LDH
Cytotoxicity Assay Kit was obtained from Thermo Fisher Scientific (Waltham, MA, USA). Filipin III were obtained from Sigma-Aldrich (St. Louis, MO, USA). Cyclodextrins (CDs) were obtained from Cyclolab (Budapest, Hungary). These CDs include $\alpha C D, \beta C D$, and $\gamma C D$ of native forms as well as their derivatives with hydroxypropyl (HP), randomly methylated (RM), and carboxymethyl (CM) substitutions. The average degree of substitution (DS) for each $\mathrm{CD}$ derivative is as follows: $\mathrm{HP} \alpha \mathrm{CD}, \mathrm{DS}=4.5$; $\mathrm{HP} \beta \mathrm{CD}, \mathrm{DS}=6 ; \mathrm{HP} \gamma \mathrm{CD}, \mathrm{DS}=4.5 ; \mathrm{RM} \alpha \mathrm{CD}, \mathrm{DS}=11$; $\mathrm{RM} \beta \mathrm{CD}, \mathrm{DS}=12 ; \mathrm{RM} \gamma \mathrm{CD}, \mathrm{DS}=12 ; \mathrm{CM} \alpha \mathrm{CD}, \mathrm{DS}=3.5$; $\mathrm{CM} \beta \mathrm{CD}, \mathrm{DS}=3.5 ; \mathrm{CM} \gamma \mathrm{CD}, \mathrm{DS}=3.5$.

\section{Cell culture and cell lines}

Untransformed skin fibroblasts from a healthy control subject (No. GM05659) or skin fibroblasts from a patient with Niemann-Pick disease type C1 (NPC1, compound heterozygote for mutations P237S and I1061T, No. GM03123) were purchased from Coriell Institute Cell Repositories (Camden, New Jersey, USA). Cells were grown in a monolayer and maintained in DMEM (with high glucose, L-glutamine, and sodium pyruvate) containing non-essential amino acids, $10 \% \mathrm{FBS}, 100 \mathrm{U} / \mathrm{ml}$ of penicillin, and $100 \mu \mathrm{g} / \mathrm{ml}$ of streptomycin at $37^{\circ} \mathrm{C}$ in a $5 \%$ $\mathrm{CO}_{2}$ humidified incubator.

Human embryo kidney cell line HEK293T and human cervical cancer-derived cell line HeLa were obtained from the American Type Culture Collection (Manassas, VA, USA). Both cell lines were grown in a monolayer and maintained in DMEM (with high glucose, L-glutamine, and sodium pyruvate) supplemented with $10 \%$ heatinactivated FBS, $100 \mathrm{U} / \mathrm{ml}$ of penicillin, and $100 \mu \mathrm{g} / \mathrm{ml}$ of streptomycin at $37^{\circ} \mathrm{C}$ in a $5 \% \mathrm{CO}_{2}$ humidified incubator.

\section{Cell viability assay}

The effect of CD treatment on cell viability or cytotoxicity were determined using the CellTiter 96 AQueous One Solution Cell Proliferation Assay Kit (Promega, Madison, WI, USA). Briefly, cells were seeded overnight in 96-well plates at a density of $1-3 \times 10^{4}$ cells per well. Cells were treated with a series of dilutions of the CDs. Cells treated with phosphate buffered saline (PBS) and cells treated with Triton X-100 (0.1\%) were included as negative and positive controls, respectively. After $24-48 \mathrm{~h}$ incubation at $37^{\circ} \mathrm{C}$, cell viability assay was performed using CellTiter 96 AQueous One Solution Cell Proliferation Assay (Promega), which is based on the conversion of the tetrazolium compound [3(4,5-dimethylthiazol-2-yl)-5-(3-carboxymethoxyphenyl)-2(4-sulfophenyl)-2H-tetrazolium, inner salt; MTS] as per the manufacturer's instructions. The formation of colored formazan product by dehydrogenase enzymes in metabolically active cells was recorded at $490 \mathrm{~nm}$ absorbance using xMark microplate absorbance spectrophotometer (Bio-Rad, 
Hercules, NJ, USA). The data were normalized to the values for untreated control. Cytotoxicity in HeLa cells was performed by Lactate dehydrogenase assay using the LDH Cytotoxicity Assay Kit (Thermo Fisher Scientific, Waltham, MA, USA).

\section{Measurement of intracellular distribution of cholesterol}

Cells were incubated in medium containing $\mathrm{CD}$ derivatives at $1 \mathrm{mM}$ for $72 \mathrm{~h}$. Cells were washed three times with PBS and fixed with $3.7 \%$ paraformaldehyde for $30 \mathrm{~min}$ at room temperature. After washing with PBS three times, cells were stained with Filipin III $(12.5 \mu \mathrm{g} / \mathrm{ml}$ in PBS) for $45 \mathrm{~min}$. at room temperature. After washing three times with PBS, cells were mounted in anti-fade mounting medium and images were taken using Nikon fluorescence microscope. The cholesterol accumulation was induced in vitro by treating HeLa cells with U18666A ( $5 \mu \mathrm{g} / \mathrm{ml}$; Enzo, Farmingdale, NY, USA) for $48-72 \mathrm{~h}$.

\section{Proteomics MudPIT analysis}

The NPC1 mutant cells or healthy donor cells treated with $\mathrm{HP} \beta C \mathrm{CD}$ or $\mathrm{HP \gamma CD}(1 \mathrm{mM}$ for $72 \mathrm{~h})$ were lysed in the lysis buffer $(20 \mathrm{mM}$ Tris-HCl, $\mathrm{pH}$ 8.0; 0.1\% Triton X-100; $100 \mu \mathrm{g} / \mathrm{ml}$ aprotinin and $100 \mu \mathrm{M}$ leupeptin protease inhibitors). The cell lysate was centrifuged at $10,000 \times g$ for $15 \mathrm{~min}$. Approximately $50 \mu \mathrm{g}$ total protein was denatured in $8 \mathrm{M}$ urea and $50 \mathrm{mM}$ Tris- $\mathrm{HCl}, \mathrm{pH} 8.0$, reduced with $10 \mathrm{mM}$ TCEP for $60 \mathrm{~min}$, alkylated with $50 \mathrm{mM}$ iodoacetamide for $60 \mathrm{~min}$, and then diluted with water to $2 \mathrm{M}$ urea and $50 \mathrm{mM}$ Tris- $\mathrm{HCl}, \mathrm{pH} 8.0$. Two micrograms of trypsin (Promega) were added for overnight digestion $(18 \mathrm{~h})$, and then the tryptic peptides were desalted using Pierce C18 spin columns (Thermo Fischer Scientific).

Desalted samples were dried in Speed-Vac, resuspended in $5 \mu \mathrm{l}$ of $0.5 \%$ formic acid and loaded onto a 3-phase MudPIT column as described previously ${ }^{42}$. A 10-step MudPIT was executed for LC-MS analysis using an Eksigent $^{\mathrm{Tm}}$ AS-1 autosampler and an Eksigent ${ }^{\mathrm{mm}} 1 \mathrm{D}$ Plus nano-LC pump online with an Orbitrap LTQ XL linear ion trap mass spectrometer (Thermo Finnigan) with a nanospray source. MS data acquisition was done in a data-dependent 6-event method (a survey FTMS scan (res. 30,000) followed by five data-dependent IT scans for five consequent most abundant ions). Database searches were done with PEAKS 8.0 and MyriMatch software packs against the forward and reverse human trypsin sequences (as downloaded from GenBank). The parameters for database search were: full tryptic digestion; up to 2 missed cleavage sites; $10 \mathrm{ppm}$ for peptide mass tolerance; $0.5 \mathrm{Da}$ for fragment mass tolerance; cysteine carbamidomethylation (+57 Da) as fixed modification; methionine oxidation $(+16 \mathrm{Da})$ as variable modification. The relative quantification of the identified proteins was performed with the $\mathrm{Q}$ module of the PEAKS software pack based on the extracted ion currents of the identified unique peptides' parent ions.

\section{Overexpression and gene silencing}

HeLa cells in monolayer were transfected with lentiviral construct using jetPrime (Polyplus Transfection, Illkirch, France) according to manufacturer's guidelines. LAMP-1 expression construct pCMV6-AC-GFP-LAMP-1 (RG219208), LAMP-1 knock down plasmid pGFP-CshLenti (TL311795), and scrambled shRNA plasmid (TR30021) were purchased from Origene (Rockville, MD, USA). Briefly, two million HeLa cells in monolayer was transfected with $1 \mu \mathrm{g}$ of plasmid and transfected cells overexpressing LAMP-1 or shRNA for LAMP-1 were selected in G418 $(50 \mu \mathrm{g} / \mathrm{ml})$ or puromycin $(5 \mu \mathrm{g} / \mathrm{ml})$, respectively. Transfection of HeLa cells was verified by the expression of the reporter protein, green fluorescence protein (GFP), using florescence microscope as well as by immunoblotting using antibodies against human LAMP-1 (D401S; Cell Signaling Technology, Danvers, MA, USA).

\section{Immunoblotting}

Cells were washed with cold PBS, and then lysed with lysis buffer with protease inhibitors as described before. The lysate was centrifuged at $10,000 \times g$ for $15 \mathrm{~min}$ at $4{ }^{\circ} \mathrm{C}$. The resulting supernatant was transferred to a new tube and protein was measured by Bradford assay. Total protein $(25 \mu \mathrm{g})$ was resolved by SDS-PAGE and transferred to nitrocellulose membrane (Bio-Rad). The membrane was blocked in 5\% skim milk for $45 \mathrm{~min}$ at room temperature followed by probing with primary antibodies overnight. After three washes with TBST $(50 \mathrm{mM}$ Tris-Cl, $150 \mathrm{mM}$ $\mathrm{NaCl}$, and $0.1 \%$ Tween 20; $\mathrm{pH} 7.6$ ), membrane was incubated with horseradish peroxidase (HRP)-conjugated secondary antibody for $2 \mathrm{~h}$ at room temperature and washed three times. Mouse anti-human LAMP-1 (D401S; Cell Signaling Technology) or rabbit anti-human LAMP-2 (PA1655, Thermo Fischer Scientific) or rabbit anti-human LAMP-3 (PA529566, Thermo Fischer Scientific) antibodies were used at 1:1000. The HRP-conjugated antimouse or anti-rabbit secondary antibodies (R\&D systems, Minneapolis, MN, USA) were used at 1:1000. The luminescent signal was developed using Super signal substrate (Thermo Fischer Scientific) and images were captured using Gel-doc system (Bio-Rad).

\section{Immunochemistry}

Cells were fixed with 3.7\% paraformaldehyde in PBS for $30 \mathrm{~min}$, washed twice with PBS, permeabilized and blocked for $30 \mathrm{~min}$ in $0.1 \%$ Triton X-100 (v/v)/1\% bovine serum albumin (w/v) in PBS. All steps were conducted at room temperature. Permeabilized cells were incubated with primary antibodies at $4{ }^{\circ} \mathrm{C}$ for overnight followed by three washes with PBS and incubation with secondary 
antibodies for $2 \mathrm{~h}$ at room temperature and three more washes. Nuclei were stained with $2 \mu \mathrm{g} / \mathrm{ml}$ of Höechst 33342 (Thermo Fischer Scientific) for $5 \mathrm{~min}$ at room temperature followed by three washes and mounting. Mouse anti-human LAMP-1 (D401S; Cell Signaling Technology), rabbit anti-human LAMP-2 (PA1655, Thermo Fischer Scientific), and rabbit anti-human LAMP-3 (PA529566, Thermo Fischer Scientific) antibodies were used at 1:100. The fluorophore-conjugated CF488A goat anti-mouse or CF594 anti-rabbit secondary antibodies (Biotium, Fremont, CA, USA) were used at 1:1000. Images were acquired using a Nikon TE2000 wide field microscope with standard filter sets using $\times 10$ objective and analyzed using Nikon image software. The distribution of lysosomes as a function of distance from the nucleus was determined by calculating the intensity of LAMP-1 fluorescence $\times$ area covered/number of nuclei in the field.

\section{Statistical analysis}

Results are expressed as mean \pm standard error of mean (S.E.M.). For comparisons, the statistical significance of differences in mean values was determined by analysis of variance (ANOVA) using GraphPad Prism 7 (GraphPad software, La Jolla, CA, USA). A $p$-value of 0.05 or less was considered statistically significant.

\section{Acknowledgements}

LC-MS proteomic analysis was conducted at the Meharry Proteomics Core at Meharry Medical College. This study was supported by the Startup Fund, the Pilot Program Grant, and the Seed Grant from Meharry Medical College awarded to BS.

\section{Author details}

'Department of Microbiology, Immunology, and Physiology, Meharry Medical College, Nashville, TN 37208, USA. ${ }^{2}$ Cyclolab Cyclodextrin Research and Development Laboratory Ltd., H-1097 Budapest, Hungary. ${ }^{3}$ Department of Internal Medicine, Meharry Medical College, Nashville, TN 37208, USA

\section{Conflict of interest}

The authors declare that they have no conflict of interest.

\section{Publisher's note}

Springer Nature remains neutral with regard to jurisdictional claims in published maps and institutional affiliations.

Supplementary Information accompanies this paper at (https://doi.org/ 10.1038/s41419-018-1056-1).

Received: 18 June 2018 Revised: 11 September 2018 Accepted: 12 September 2018

Published online: 03 October 2018

\section{References}

1. Goldstein, J. L., Dana, S. E., Faust, J. R., Beaudet, A. L. \& Brown, M. S. Role of lysosomal acid lipase in the metabolism of plasma low density lipoprotein. Observations in cultured fibroblasts from a patient with cholesteryl ester storage disease. J. Biol. Chem. 250, 8487-8495 (1975).
2. Demel, R. A. \& De Kruyff, B. The function of sterols in membranes. Biochim Biophys. Acta 457, 109-132 (1976).

3. Simons, K. \& Ikonen, E. How cells handle cholesterol. Science 290, 1721-1726 (2000).

4. Goldstein, J. L., DeBose-Boyd, R. A. \& Brown, M. S. Protein sensors for membrane sterols. Cell 124, 35-46 (2006).

5. Rosenbaum, A. I. \& Maxfield, F. R. Niemann-Pick type C disease: molecular mechanisms and potential therapeutic approaches. J. Neurochem. 116, 789-795 (2011).

6. Sleat, D. E. et al. Genetic evidence for nonredundant functional cooperativity between NPC1 and NPC2 in lipid transport. Proc. Natl Acad. Sci. USA 101, 5886-5891 (2004)

7. Davis, M. E. \& Brewster, M. E. Cyclodextrin-based pharmaceutics: past, present and future. Nat. Rev. Drug Discov. 3, 1023-1035 (2004).

8. Abi-Mosleh, L, Infante, R. E, Radhakrishnan, A., Goldstein, J. L. \& Brown, M. S. Cyclodextrin overcomes deficient lysosome-to-endoplasmic reticulum transport of cholesterol in Niemann-Pick type C cells. Proc. Natl Acad. Sci. USA 106, 19316-19321 (2009).

9. Davidson, C. D. et al. Chronic cyclodextrin treatment of murine Niemann-Pick C disease ameliorates neuronal cholesterol and glycosphingolipid storage and disease progression. PLOS ONE 4, e6951 (2009).

10. Camargo, F. et al. Cyclodextrins in the treatment of a mouse model of Niemann-Pick C disease. Life Sci. 70, 131-142 (2001).

11. Ramirez, C. M. et al. Weekly cyclodextrin administration normalizes cholesterol metabolism in nearly every organ of the Niemann-Pick type C1 mouse and markedly prolongs life. Pediatr. Res. 68, 309-315 (2010).

12. Vite, C. H. et al. Intracisternal cyclodextrin prevents cerebellar dysfunction and Purkinje cell death in feline Niemann-Pick type C1 disease. Sci. Transl. Med. 7, $276 r a 226$ (2015)

13. Maarup, T. J. et al. Intrathecal 2-hydroxypropyl-beta-cyclodextrin in a single patient with Niemann-Pick C1. Mol. Genet. Metab. 116, 75-79 (2015).

14. Ory, D. S. et al. Intrathecal 2-hydroxypropyl-beta-cyclodextrin decreases neurological disease progression in Niemann-Pick disease, type C1: a non-randomised, open-label, phase 1-2 trial. Lancet 390, 1758-1768 (2017).

15. Soga, M. et al. HPGCD outperforms HPBCD as a potential treatment for Niemann-Pick disease type $C$ during disease modeling with iPS cells. Stem Cells 33, 1075-1088 (2015)

16. Dai, S. et al. Methyl-beta-cyclodextrin restores impaired autophagy flux in Niemann-Pick C1-deficient cells through activation of AMPK. Autophagy 13, 1435-1451 (2017)

17. Szente, L., Singhal, A., Domokos, A. \& Song, B. Cyclodextrins: assessing the impact of cavity size, occupancy, and substitutions on cytotoxicity and cholesterol homeostasis. Molecules 23, E1228 (2018).

18. Wolters, D. A., Washburn, M. P. \& Yates, J. R. 3rd An automated multidimensional protein identification technology for shotgun proteomics. Anal. Chem. 73, 5683-5690 (2001).

19. Mueller, L. N. et al. SuperHirn - a novel tool for high resolution LC-MS-based peptide/protein profiling. Proteomics 7, 3470-3480 (2007).

20. Kislinger, T. \& Emili, A. Multidimensional protein identification technology: current status and future prospects. Expert Rev. Proteom. 2, 27-39 (2005).

21. Ross, P. L. et al. Multiplexed protein quantitation in Saccharomyces cerevisiae using amine-reactive isobaric tagging reagents. Mol. Cell Proteom. 3, 1154-1169 (2004)

22. Washburn, M. P., Ulaszek, R. R. \& Yates, J. R. 3rd Reproducibility of quantitative proteomic analyses of complex biological mixtures by multidimensional protein identification technology. Anal. Chem. 75, 5054-5061 (2003).

23. Lebrand, C. et al. Late endosome motility depends on lipids via the small GTPase Rab7. EMBO J. 21, 1289-1300 (2002).

24. Ko, D. C., Gordon, M. D., Jin, J. Y. \& Scott, M. P. Dynamic movements of organelles containing Niemann-Pick C1 protein: NPC1 involvement in late endocytic events. Mol. Biol. Cell 12, 601-614 (2001).

25. Zhang, M. et al. Cessation of rapid late endosomal tubulovesicular trafficking in Niemann-Pick type C1 disease. Proc. Natl Acad. Sci. USA 98, 4466-4471 (2001).

26. Ottinger, E. A. et al. Collaborative development of 2-hydroxypropyl-betacyclodextrin for the treatment of Niemann-Pick type C1 disease. Curr. Top. Med Chem. 14, 330-339 (2014).

27. Matsuo, M. et al. Effects of cyclodextrin in two patients with Niemann-Pick Type C disease. Mol. Genet. Metab. 108, 76-81 (2013).

28. Irie, T., Fukunaga, K. \& Pitha, J. Hydroxypropylcyclodextrins in parenteral use. I: Lipid dissolution and effects on lipid transfers in vitro. J. Pharm. Sci. 81 521-523 (1992). 
29. Eskelinen, E. L., Tanaka, Y. \& Saftig, P. At the acidic edge: emerging functions for lysosomal membrane proteins. Trends Cell Biol. 13, 137-145 (2003).

30. Wilke, S., Krausze, J. \& Bussow, K. Crystal structure of the conserved domain of the DC lysosomal associated membrane protein: implications for the lysosomal glycocalyx. BMC Biol. 10, 62 (2012).

31. Kundra, R. \& Kornfeld, S. Asparagine-linked oligosaccharides protect Lamp-1 and Lamp-2 from intracellular proteolysis. J. Biol. Chem. 274, 31039-31046 (1999).

32. Granger, B. L. et al. Characterization and cloning oflgp110, a lysosomal membrane glycoprotein from mouse and rat cells. J. Biol. Chem. 265, 12036-12043 (1990).

33. Huynh, K. K. et al. LAMP proteins are required for fusion of lysosomes with phagosomes. EMBO J. 26, 313-324 (2007).

34. Eskelinen, E. L. et al. Disturbed cholesterol traffic but normal proteolytic function in LAMP-1/LAMP-2 double-deficient fibroblasts. Mol. Biol. Cell 15 3132-3145 (2004).

35. Schneede, A. et al. Role for LAMP-2 in endosomal cholesterol transport. J. Cell Mol. Med. 15, 280-295 (2011).
36. Hulce, J. J., Cognetta, A. B., Niphakis, M. J., Tully, S. E. \& Cravatt, B. F. Proteomewide mapping of cholesterol-interacting proteins in mammalian cells. Nat Methods 10, 259-264 (2013).

37. Li, J. \& Pfeffer, S. R. Lysosomal membrane glycoproteins bind cholesterol and contribute to lysosomal cholesterol export. Elife 5, e21635 (2016).

38. Xu, S., Benoff, B., Liou, H. L., Lobel, P. \& Stock, A. M. Structural basis of stero binding by NPC2, a lysosomal protein deficient in Niemann-Pick type C2 disease. J. Biol. Chem. 282, 23525-23531 (2007).

39. Infante, R. E. et al. Purified NPC1 protein. I. Binding of cholesterol and oxysterols to a 1278-amino acid membrane protein. J. Biol. Chem. 283, 1052-1063 (2008).

40. Naureckiene, S. et al. Identification of HE1 as the second gene of NiemannPick C disease. Science 290, 2298-2301 (2000).

41. Pentchev, P. G. Niemann-Pick $C$ research from mouse to gene. Biochim Biophys. Acta 3-7, 2004 (1685).

42. Link, A. J. et al. Direct analysis of protein complexes using mass spectrometry. Nat. Biotechnol. 17, 676-682 (1999). 